\title{
Enzyme-linked Immunosorbent Assay to Detect Urinary Antibody Against Recombinant rKRP42 Antigen Made from Leishmania donovani for the Diagnosis of Visceral Leishmaniasis
}

\author{
Mohammad Zahidul Islam,* Makoto Itoh, Hidekazu Takagi, Anwar Ul Islam, A. R. M. Saifuddin Ekram, \\ Ajijur Rahman, Atsuhide Takesue, Yoshihisa Hashiguchi, and Eisaku Kimura \\ Department of Parasitology, Aichi Medical University School of Medicine, Nagakute, Aichi, Japan; Department of Pharmacy, \\ University of Rajshahi, Rajshahi, Bangladesh; Department of Medicine, Rajshahi Medical College, Rajshahi, Bangladesh; \\ Department of Parasitology, Kochi Medical School, Kochi University, Nankoku, Japan
}

\begin{abstract}
We recently reported the production of the recombinant kinesin-related protein of Leishmania donovani with a molecular weight of $42 \mathrm{kd}$ (rKRP42) and the value of the antigen in serum-based ELISA for the diagnosis of visceral leishmaniasis (VL). In this study, the rKRP42 antigen was validated with ELISA using urine samples (rKRP42 urine ELISA). The urine-based ELISA showed 94\% sensitivity (108 positives among 115 VL samples) and $99.6 \%$ specificity (239 negatives among 240 non-VL samples). The sensitivity and specificity are almost similar to our previous results by ELISA with acetone-treated $L$. donovani promastigote antigen and direct agglutination test, both methods being done by use of urine samples. A comparison of the rKRP42 urine ELISA with the commercially available urinary antigen detection kit (KAtex) using 108 VL samples showed much higher sensitivity of the ELISA (96.3\%) than KAtex $(55.6 \%)$. The use of the rKRP42 antigen with urine samples will facilitate epidemiologic studies.
\end{abstract}

\section{INTRODUCTION}

Visceral leishmaniasis (VL) is caused by a protozoan parasite Leishmania donovani complex. In full-blown disease, the mortality rate ranges from $80 \%$ to $100 \%$ if not treated. Even with treatment, sometimes the case fatality rate reaches $>10 \% .^{1}$ Recently, in a population-based study in Bangladesh, Ahlwalia and others ${ }^{2}$ reported a VL incidence of $2 \%$ per year from 2000 to 2002 , with a case fatality rate of $19 \%$ among adult women ( $\geq 15$ years of age) and $6-8 \%$ among the other demographic groups (girls $<15$ years of age and males of all ages). They also reported that the median duration of illness before receiving treatment was significantly longer in females than males. Delays in diagnosis and treatment increase the risk of complications and death. ${ }^{1} \mathrm{VL}$ is targeted for control under the neglected tropical diseases program. ${ }^{3}$ The effective control of VL has been hampered because of the lack of suitable tools for early detection of infection and effective chemotherapy. Therefore, an accurate, easy, and low-cost diagnostic method is an urgent necessity.

Because of invasiveness and inadequate sensitivities, demonstration of the causative parasites in aspirates from lymph nodes, bone marrow, and the spleen has limited its use in a field survey. ${ }^{4}$ In the last two decades, several serodiagnoses, like ELISA with crude or recombinant antigens, ${ }^{5-7}$ and direct agglutinin test (DAT), ${ }^{8,9}$ have been providing good diagnostic results. However, the use in endemic areas has been limited because of the expertise required for execution. In Bangladesh, DAT only has been available for clinicians at the Institute of Epidemiology, Disease Control and Research (IEDCR) situated in the capital, Dhaka. Recently, a recombinant antigen rK39, a part of L. chagasi kinesin-related protein, has been widely evaluated with ELISA or in dipstick format. ${ }^{10,11}$ Although the antigen has been reported satisfactory in Indian subcontinent, the results varied considerably in different endemic areas. In India and Nepal, ${ }^{12-14}$ the test

* Address correspondence to Mohammad Zahidul Islam, Department of Parasitology, Aichi Medical University School of Medicine, Nagakute, Aichi-ken 480-1195, Japan, 5. E-mail: islammz@yahoo.com showed the highest sensitivity of $100 \%$, whereas it was significantly lower in Venezuela $(88 \%),{ }^{15}$ in southern Europe $(71.4 \%),{ }^{16}$ and in Sudan $(67 \%) .{ }^{17}$ The variation could be explained by differences in subspecies of $L$. donovani complexes, genetic differences in individual patients or in racial subgroups, and epidemiologic factors such as the length or severity of diseases. ${ }^{18}$ Thus, it is desirable to develop new antigens for comparison. Recently, we reported the production of recombinant protein $\mathrm{rKRP} 42$, a part of $L$. donovani kinesin-related protein and a homolog to rK39, and evaluation of this antigen in ELISA with serum samples for the diagnosis of VL. ${ }^{19}$

Recently, the use of urine for blood has been considered valuable for the diagnosis of VL. A low-molecular-weight heat-stable leishmanial carbohydrate antigen was detected in urine by a latex agglutination test (KAtex). ${ }^{20,21}$ We reported an ELISA with urine samples using acetone-treated L. donovani promastigotes antigen (acetone-treated urine ELISA), ${ }^{22}$ and a direct agglutination test with urine samples (urine DAT $)^{23}$ for the diagnosis of VL, which showed, respectively, sensitivities of $95 \%$ and $90.7 \%$ and specificities of $95.3 \%$ and $97.3 \%$. These figures are almost comparable to serum-based ELISA and DAT. In this study, we report the usefulness of rKRP42 antigen in a new urine ELISA and compare the results with other urine-based immunologic tests.

\section{MATERIALS AND METHODS}

Urine and serum samples. Most urine samples tested in this study were the same ones used in our previous study. ${ }^{23} \mathrm{Ad}$ ditional samples from 44 VL patients and 17 Japanese healthy controls were included. A total of 115 urine samples from defined VL patients, collected from different medical college hospitals (96 from Rajshahi Medical College hospital, 11 from Mymensingh Medical College hospital, and 8 from Institute of Epidemiology, Disease Control and Research) in Bangladesh, were used to compute sensitivity of rKRP42 urine ELISA. VL was diagnosed in accordance with the WHO guideline of initiation of treatment. ${ }^{24}$ Briefly, the diagnosis was based on well-defined clinical symptoms along with sup- 
portive hematologic features (anemia, leucopenia, reversed albumin globulin ratio, etc.) and was confirmed by parasitologic test or at least by one serologic test. The clinical symptoms included at least three of the following symptoms: intermittent chronic fever for $>1$ month, splenomegaly, hepatomegaly, anemia, wasting, and lymphadenopathy. Malaria, tuberculosis, enteric fever, and other diseases that could produce similar clinical symptoms had been ruled out as much as possible. Among the 115 patients, 40 were confirmed parasitologically: Leishman-Donovan bodies were detected in smears of splenic aspirates (30 patients) or bone marrow aspirates ( 5 patients), and promastigotes were shown in 5 patients after inoculation of aspirate materials in Novy, MacNeal, and Nicolle medium. Of the other 75 clinically confirmed patients, 47, 9, and 19 were positive by conventional DAT, aldehyde test, and rK39 dipstick test, respectively. At the time of sample collection, patients were either in the course of treatment with sodium antimony gluconate at a WHO-recommended dose ${ }^{25}$ or just before the initiation of treatment. A total of 240 non-VL urine samples were used for a specificity study. Fifty-nine control samples were taken from apparently healthy individuals with no past history of kalaazar from endemic areas in Bangladesh (endemic healthy control [EHC]). Eighty samples from Japanese individuals were used as non-endemic healthy controls (NEHC). Fiftyeight malaria samples collected in Solomon Islands, 13 tuberculosis samples from Bangladesh, 23 cutaneous leishmaniasis (CL) samples from Ecuador, and 7 samples from patients with other diseases were also included. Other diseases category included amebic liver abscess (2 cases), aplastic anemia (2), aplastic anemia with nephrotic syndrome (1), aortic stenosis (1), and viral fever (1). Immediately after collection of urine, $\mathrm{NaN}_{3}$ was added to each sample at the final concentration of $0.1 \%$ as preservative, and the samples were transported to Japan at ambient temperature and kept at $4^{\circ} \mathrm{C}$ until immunologic tests.

The study was reviewed and approved by the Ethics Committee of Aichi Medical University School of Medicine, Japan, and the Ethical Review Committee of the Bangladesh Medical Research Council.

Effect of storage of urine samples on $L$. donovani-specific IgG titers. This study included urine samples that had been collected $>6$ years before. To study the effect of storage of $\mathrm{NaN} 3$-added urine samples at $4^{\circ} \mathrm{C}$, L. donovani-specific IgG titers of the same urine samples from $51 \mathrm{VL}$ and 211 non-VL subjects were measured with acetone-treated urine ELISA at three different times: the first measurement with relatively fresh urine samples (collected within 1 year) was in February 2001, the second one in May 2003, and the third one in November 2007. The ELISA with acetone-treated $L$. donovani promastigotes antigen was described elsewhere. ${ }^{22}$ The measurement (all in duplicate) was standardized using a set of serially diluted positive sera prepared for every microtiter plate. The sensitivities and specificities obtained in the three measurements were compared using the $\chi^{2}$ test, and the geometric mean IgG titers were compared using paired $t$ test.

The rKRP42 urine ELISA. The production of rKRP42 antigen was described elsewhere. ${ }^{19}$ An ELISA was performed as follows. Flat-bottomed 96-well microtiter plates (MaxiSorp; Nunc, Roskilde, Denmark) were coated with $1 \mu \mathrm{g} / \mathrm{mL}$ (100 $\mu \mathrm{L} /$ well) rKRP42 antigen and incubated overnight at $4{ }^{\circ} \mathrm{C}$. After blocking with casein buffer $(1 \%$ casein in 0.05
mol/L Tris- $\mathrm{HCl}$ buffer with $0.15 \mathrm{~mol} / \mathrm{L} \mathrm{NaCl}, \mathrm{pH} 7.6$ ) for 2 hours at room temperature, the wells were loaded with 100 $\mu \mathrm{L}$ of urine without concentration and incubated at $37^{\circ} \mathrm{C}$ for 1 hour. After four washes with PBS(-) (pH 7.4) containing $0.05 \%$ Tween 20, peroxidase-conjugated goat anti-human IgG (Tago, Camarillo, CA), diluted 1:4,000 with casein buffer, was added and incubated at $37^{\circ} \mathrm{C}$ for 1 hour. After washing four times, ABTS substrate (KPL, Gaithersburg, MD) was reacted for coloration for 1 hour at room temperature. The optical density was measured at 415 and $492 \mathrm{~nm}$ as reference. Each sample was assayed in duplicate, in which, if the absorbance values of the duplicates differ $>40 \%$ from their average, the sample was retested. Antibody levels were expressed arbitrarily as unit (U), which was estimated from the standard curve constructed for each plate with serially diluted positive sera. The cut-off point for anti-rKRP42 IgG was first calculated as the mean plus 3 SDs of $\log$ (unit +1 ) values of the $\mathrm{NEHC}$, and [antilog of (the mean +3 SDs) -1 ] was regarded as the cut-off unit.

Urine latex agglutination test (KAtex). The KAtex was performed with $108 \mathrm{VL}$ (7 VL samples could not be tested because of inadequate quantity) and 240 non-VL urine samples according to the manufacturer's protocol (Kalon Biological, Aldershot, UK) except that $100 \mu \mathrm{L}$ of urine was used for pretreatment of 5-minute boiling instead of recommended $250 \mu \mathrm{L}$ to $1 \mathrm{~mL}$. Before changing the volume of urine, we tested 10 urine samples and confirmed that the use of $100 \mu \mathrm{L}$ did not influence the results. After bringing all the test reagents to ambient temperature, $50 \mu \mathrm{L}$ of urine sample was placed to a reaction zone in the glass slide, and a drop of test latex was added to it. The liquids were stirred to a completely homogenous mixture and rotated continuously for 2 minutes. A negative control in the reaction zone next to the test sample was run each time. Any agglutination discerned compared with the negative control was considered positive.

\section{RESULTS}

Effect of storage of urine samples. Fifty-six VL and 211 non-VL urine samples stored at $4^{\circ} \mathrm{C}$ were tested with the acetone-treated urine ELISA in February 2001, May 2003, and November 2007, which showed sensitivities of $91.1 \%$, $91.1 \%$, and $82.1 \%$ and specificities of $95.3 \%, 97.2 \%$, and $98.1 \%$, in order of measurement (Table 1). There were no differences among the sensitivities ( $\chi^{2}$ test, $\left.2 \mathrm{df}, P>0.24\right)$ and the specificities ( $\chi^{2}$ test, $2 \mathrm{df}, P>0.23$ ). The mean $\mathrm{IgG}$ units in the first, second, and third measurements with the VL samples were $103.10,83.86$, and $61.11 \mathrm{U}$, respectively, and those with the non-VL samples were $0.93,0.94$, and $0.89 \mathrm{U}$, respectively (Table 1 ). In the VL samples, paired $t$ test showed significant differences between the first and second means $(P<0.012)$, the second and third means $(P<0.002)$, and the first and third means $(P<0.001)$. Because the first measurement was done with relatively fresh samples, we considered its mean unit as $100 \%$ and calculated the percentages of IgG units in the second and third measurements. They were $81.3 \%$ and $59.3 \%$, respectively (Table 1 ). The percentage reduction, when plotted on a graph, showed an approximate linearity, and a yearly rate of reduction was estimated to be $6.0 \%$ (40.7\% reduction in 6.8 years). In the non-VL samples, no significant differences were obtained $(P>0.28$ in all comparisons). 
TABLE 1

Effect of storage of samples on sensitivity and specificity and on mean antibody unit with acetone treated urine ELISA

\begin{tabular}{|c|c|c|c|c|}
\hline & & \multicolumn{3}{|c|}{ Measurement time } \\
\hline & & 2001 February & 2003 May & 2007 November \\
\hline \multirow{4}{*}{ Mean antibody unit (\%) } & Sensitivity & $91.1 \%(51 / 56)$ & $91.1 \%(51 / 56)$ & $82.1 \%(46 / 56)$ \\
\hline & Specificity & $95.3 \%(201 / 211)$ & $97.2 \%(205 / 211)$ & $98.1 \%(207 / 211)$ \\
\hline & VL & $103.10(100.0)$ & $83.86(81.3)$ & $61.11(59.3)$ \\
\hline & Non-VL & $0.93(100.0)$ & 0.94 (101.2) & $0.89(95.5)$ \\
\hline
\end{tabular}

The rKRP42 Urine ELISA. A total of $115 \mathrm{VL}$ and 240 non-VL samples were tested for IgG using rKRP42 antigen (Figure 1). With the NEHC group, the cut-off was determined as $57.9 \mathrm{U}$, based on which the test showed a sensitivity of 94.0\% (108 positives among 115 VL samples) and a specificity of $99.6 \%$ (239 negatives among 240 non-VL samples). Of the 108 positive VL samples, only 10 had titers $<1,000 \mathrm{U}$. There was no difference between the sensitivities when calculated with the parasite-confirmed VL group (92.5\% with 40 samples) and clinical VL group (94.7\% with 75 samples; $\chi^{2}$ test, $P>0.64)$. The specificities for EHC, NEHC, M, TB, and CL were $100 \%$. Only one sample of other diseases category (aplastic anemia with nephrotic syndrome) became positive by the test.

KAtex. The urinary antigen detection assay KAtex showed a low sensitivity of $55.6 \%$ (60 positives among $108 \mathrm{VL}$ samples) and a high specificity of $100 \%$ with 240 non-VL samples. Among the 108 VL samples, 36 were parasite positive, the rest (72) were clinical VL, and the KAtex positive rate for the former group was $63.9 \%$ (23 positives) and for the latter group was $51.4 \%$ (37 positives). No significance difference was obtained between the groups ( $\chi^{2}$ test, $\left.P>0.21\right)$. In this study, 13 samples (of which 5 were parasitologically confirmed) were collected before initiation of treatment, and the rest (95 samples) were collected at various times in the course of treatment. Further analysis showed that the pre-treatment samples had a high sensitivity of $76.9 \%$, whereas the treated samples had much lower sensitivity (44-54\%; Table 2). However, the difference between the pre- and undertreatment (all combined) samples was not significant ( $\chi^{2}$ test, $\left.P>0.09\right)$.

\section{DISCUSSION}

Because most of the urine samples used in this study were stored at $4^{\circ} \mathrm{C}$ for years, it would be essential to study the effect of storage on sensitivity, specificity, and the level of antibody unit, and for the study, acetone-treated urine

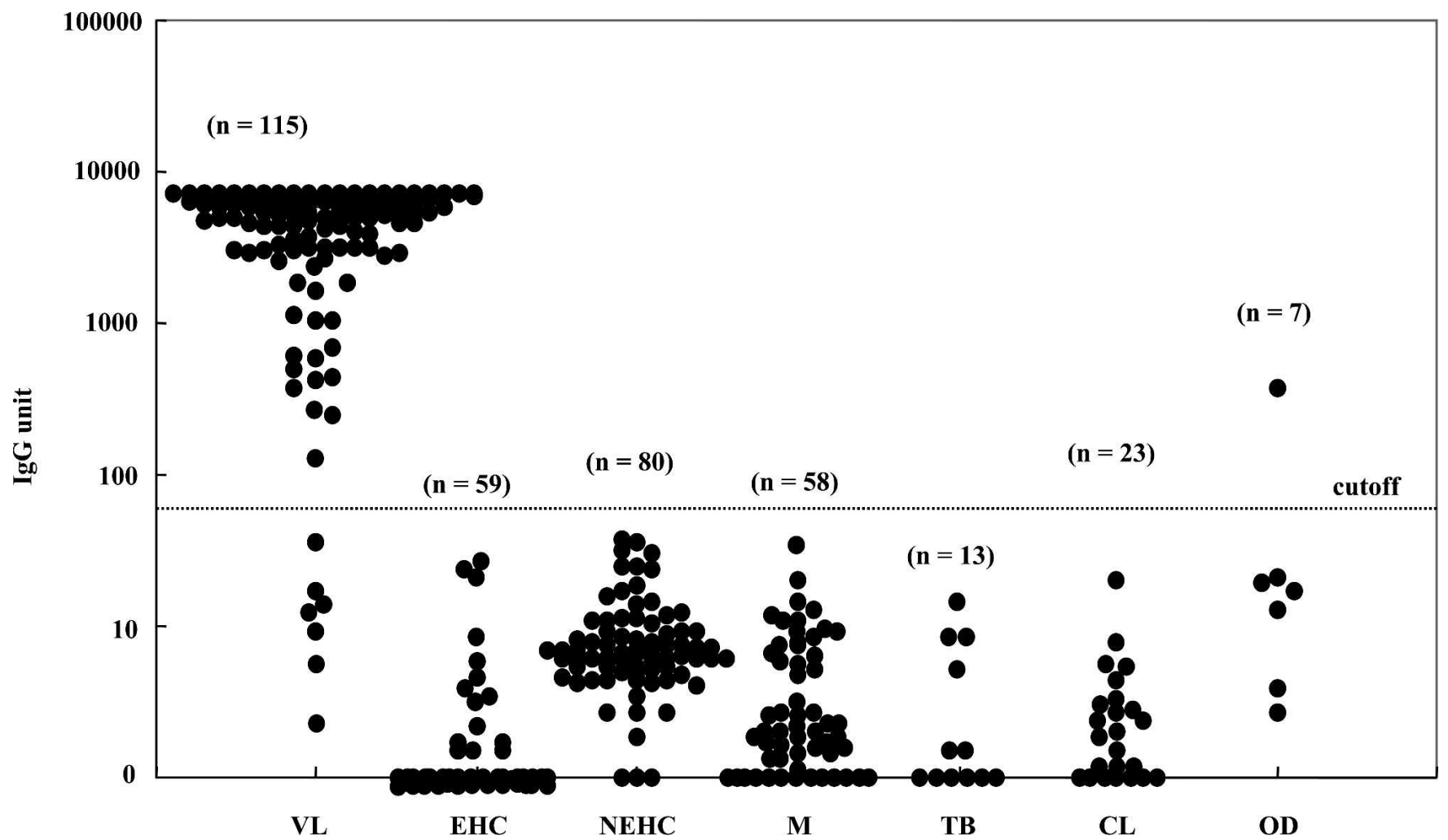

FIGURE 1. Detection of anti-rKRP42 IgG in urine of visceral leishmaniasis patients, healthy individuals, and controls with various diseases by ELISA. Each symbol $(\bullet)$ stands for a single urine sample. The horizontal dotted line represents the cut-off value: $57.9 \mathrm{U}$. VL $=$ visceral leishmaniasis; $\mathrm{EHC}=$ endemic healthy controls; $\mathrm{NEHC}=$ non-endemic healthy controls; $\mathrm{M}=$ malaria; $\mathrm{TB}=$ tuberculosis; $\mathrm{CL}=$ cutaneous leishmaniasis; OD $=$ other diseases; $n=$ number of samples. 
TABLE 2

KAtex-positive rate in relation to sample collection day with treatment

\begin{tabular}{lcl}
\hline \multicolumn{1}{c}{$\begin{array}{c}\text { Sample collected on the day after } \\
\text { initiation of treatment }\end{array}$} & $\begin{array}{c}\text { Tested } \\
\text { samples }\end{array}$ & KAtex positives \\
\hline 0 (before initiation of treatment) & 13 & $76.9 \%(10 / 13)$ \\
$1 \sim 10$ & 9 & $44.4 \%(4 / 9)$ \\
$11 \sim 20$ & 15 & $53.3 \%(8 / 15)$ \\
$21 \sim 30$ or more & 6 & $50.0 \%(3 / 6)$ \\
No clear history* & 65 & $53.8 \%(35 / 65)$ \\
* In the patient's data sheets, the exact date of collection was not mentioned, but all the \\
samples were collected within the course of treatment
\end{tabular}

ELISA was applied. The storage clearly decreased mean antibody units at a rate of $6 \%$ annually in the VL group. To the best of our knowledge, this is the first quantitative report on the reduction of $L$. donovani-specific antibody after longterm storage of $\mathrm{NaN}_{3}$-added urine samples at $4^{\circ} \mathrm{C}$. Thus, theoretically, urine storage could decrease sensitivity and at the same time might improve specificity. However, such effects will be only on borderline positive titers. In this study, up to 6 years and 8 months of storage did not affect the sensitivity and specificity at a statistically significant level.

The rKRP42 urine ELISA with samples kept for years showed a high sensitivity of $94 \%$. The ELISA also resulted in a very high specificity of $99.6 \%$. These results are comparable to our previously reported figures obtained by the acetonetreated urine ELISA, urine DAT, and rKRP42 serum ELISA. The commercially available rK39 dipstick test (InBios International, Seattle, WA) also gave similar results in our study in Bangladesh (Table 3).

When three different methods for urinary antibody detection (rKRP42 urine ELISA, acetone-treated urine ELISA, and urine DAT) were taken into consideration, a total $90.1 \%$ (64 of 71) VL samples were positive and 95.9\% (211 of 220) of non-VL samples were negative by all three tests. Variable results, i.e., positive by one test but negative by other test(s), were obtained in 3 of $71 \mathrm{VL}$ samples (4.2\%), and 8 of 220 non-VL samples (3.6\%). All these methods failed to detect four VL cases, of which three were parasite positives, or cross-reacted with one non-VL sample that was collected from a patient of aplastic anemia with nephrotic syndrome.

When we compared the rKRP42 urine ELISA with the rKRP42 serum ELISA and the rK39 dipstick test using $71 \mathrm{VL}$ cases, $65(91.5 \%)$ samples became positive and $4(4.6 \%)$ samples were negative in all assays. Two samples from one parasite-confirmed and one clinical VL gave false-negative results with rKRP42 urine ELISA but were positives by rKRP42 serum ELISA. Of the four false negatives, three were collected from parasitologically confirmed patients. These three samples gave false-negative results with conventional serum-based $\mathrm{DAT}^{23}$ and with urinary antibody detection assays (acetone-treated urine ELISA and urine DAT).

There is always a fraction of infected persons without clinical manifestations. Khalil and others ${ }^{26}$ reported that, in eastern Sudan, the ratios of clinical and subclinical cases in UmSalala village, respectively, in 1994/95 and 1995/96, were 1.2:1 and 2.6:1, and in Mashrau Koka village were 1:11 and 1:2.5. In another study conducted in Bihar state, India, $69 \%$ of asymptomatic seropositves by rK39 ELISA and dipstick developed kala-azar within 1 year, ${ }^{27}$ suggesting that many of the asymptomatic cases were in a pre-clinical state. In predicting possible clinical cases, an ELISA, which is quantitative, would be

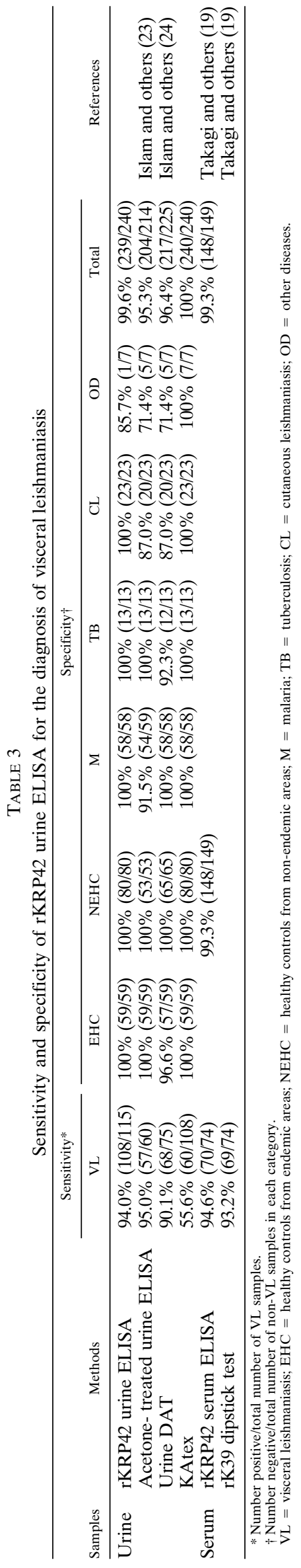


more advantageous than a dipstick format, that is, high antibody titers, or increase in antibody titers with time could be indicative of possible clinical cases. Such early diagnosis will have a practical importance now that oral treatment with miltefosine has become available. ${ }^{28}$ However, frequent collection of blood often hinders the field surveys. Urine samples that can be easily and safely collected will solve many of the difficulties experienced with blood collection in a field study, especially with children.

Antigen detection is more useful than antibody detection for diagnosing active VL cases. KAtex has been widely evaluated in many places such as in $\mathrm{Nepal}^{29}$ and India ${ }^{30}$ and found to have a sensitivity of $47.7 \%$ and $73.5 \%$, respectively. In this study, the sensitivity and specificity of KAtex was $55.6 \%$ and $100 \%$, respectively. Because the study included urine samples from clinically suspected cases and undertreatment cases, the sensitivity could be an underestimate. In our separate study, it was observed that six of seven KAtex-positive cases turned negative after completion of 28-day course of sodium stibogluconate treatment, suggesting its diagnostic and prognostic value.

The rKRP42 urine ELISA has been shown to be highly sensitive and specific. The stability of urinary antibody at $4^{\circ} \mathrm{C}$ even after prolonged storage has also been confirmed. Our rKRP42 urine ELISA would be a better alternative for the diagnosis of VL in a field survey, especially when repeated sampling is needed. KAtex that uses urine would be good adjunct for determining the active VL cases among rKRP42 urine ELISA positives.

Received June 3, 2008. Accepted for publication July 17, 2008.

Acknowledgments: We thank Drs. Rusella Mirza, Farzana Matin, Mohsin Ali, A. K. M. Shamsuzzaman Choudhury, Akram Hossain, Nilufar Begam, and Sultana Monira Hussain for helping in collection of VL samples and Dr. Judson. L. Leafasia for providing malaria samples.

Financial support: This study was supported by Grant-in-Aid for Scientific Research for JSPS Postdoctoral Fellowship for Foreign Researchers 16. 04227, Grant-in-Aid for Scientific Research (B) 15406018, and Grant-in-Aid for Scientific Research (B) 18406013 from the Japan Society for the Promotion of Science, and also by the Special Coordination Funds for Promoting Science and Technology of MEXT, Japan, 1200015.

Authors' addresses: Mohammad Zahidul Islam, Makoto Itoh, Hidekazu Takagi, Atsuhide Takesue, and Eisaku Kimura: Department of Parasitology, Aichi Medical University School of Medicine, Nagakute, Aichi-ken 480-1195, Japan, Tel: 81-52-264-4811, Fax: 81-56163-3645, E-mails: zahid@aichi-med-u.ac.jp, macitoh@aichi-medu.ac.jp, htakagi@aichi-med-u.ac.jp, atakesue@aichi-med-u.ac.jp, and kimura@aichi-med-u.ac.jp. Anwar Ul Islam and Ajijur Rahman, Department of Pharmacy, University of Rajshahi, Rajshahi, Bangladesh, Tel: 88-721-750041-9, Fax: 88-721-750064, E-mails: profanwarulislam@yahoo.com and shamim_ru2003@yahoo.com. A. R. M. Saifuddin Ekram, Department of Medicine, Rajshahi Medical College, Rajshahi, Bangladesh, Tel: 88-721-776001-9, Fax: 88-721772174, E-mail: armsekram@yahoo.com. Yoshihisa Hashiguchi, Department of Parasitology, Kochi Medical school, Kochi University, Nankoku City, Kochi 783-8505, Japan, Tel: 81-88-880-2415, Fax: 8188-880-2415, E-mail: hasiguti@kochi-u.ac.jp.

Reprint requests: Mohammad Zahidul Islam, Department of Parasitology, Aichi Medical University School of Medicine, Nagakute, Aichi-ken 480-1195, Japan, E-mails: zahid@aichi-med-u.ac.jp and islammz@yahoo.com.

\section{REFERENCES}

1. Collin S, Davidson R, Ritmeijer K, Keus K, Melaku Y, Kipngetich S, Davies C, 2004. Conflict and kala-azar: determinants of adverse outcome of kala-azar among patients in Southern Sudan. Clin Infect Dis 38: 612-619.

2. Ahluwalia IB, Bern C, Costa C, Akter T, Chowdhury R, Ali M, Alam D, Kenah E, Amann J, Islam M, Wagatsuma Y, Haque R, Breiman RF, Maguire JH, 2003. Visceral leishmaniasis: consequences of a neglected disease in a Bangladeshi community. Am J Trop Med Hyg 69: 624-628.

3. World Health Organization, 2007. Global Plan to combat Neglected Tropical Diseases, 2008-2015. Geneva, Switzerland: World Health Organization.

4. Zijlstra EE, Ali MS, el-Hassan AM, el-Toum IA, Satti M, Ghalib HW, Kager PA, 1992. Kala-azar: a comparative study of parasitological methods and the direct agglutination test in diagnosis. Trans $R$ Soc Trop Med Hyg 86: 505-507.

5. Kaul P, Malla N, Kaur S, Mahajan RC, Ganguly NK, 2000. Evaluation of a $200-\mathrm{kDa}$ amastigote-specific antigen of $L$. donovani by enzyme-linked immunosorbent assay (ELISA) for the diagnosis of visceral leishmaniasis. Trans $R$ Soc Trop Med Hyg 94: $173-175$.

6. Raj VS, Ghosh A, Dole VS, Madhubala R, Myler PJ, Stuart KD, 1999. Serodiagnosis of leishmaniasis with recombinant ORFF antigen. Am J Trop Med Hyg 61: 482-487.

7. Zijlstra EE, Daifalla NS, Kager PA, Khalil EAG, El-Hassan AM, Reed SG, Ghalib HW, 1998. rK39 enzyme-linked immunosorbent assay for diagnosis of Leishmania donovani infection. Clin Diagn Lab Immunol 5: 717-720.

8. Harith AE, Kolk AHJ, Kager PA, Leeuwenburg J, Muigai R, Kiugu S, Laarman JJ, 1986. A simple and economical direct agglutination test for serodiagnosis and sero-epidemiological studies of visceral leishmaniasis. Trans $R$ Soc Trop Med Hyg 80: $583-587$.

9. Harith AE, Kolk AHJ, Leeuwenburg J, Muigai R, Huigen E, Jelsma T, Kager PA, 1988. Improvement of a direct agglutination test for field studies of visceral leishmaniasis. J Clin Microbiol 26: 1321-1325.

10. Burns JM, Shreffler WG, Benson DR, Ghalib HW, Badaro R Reed SG, 1993. Molecular characterization of a kinesin-related antigen of Leishmania chagasi that detects specific antibody in African American visceral leishmaniasis. Proc Natl Acad Sci USA 90: 775-779.

11. Maalej IA, Chenik M, Louzir H, Salah AB, Bahloul C, Amri F, Dellagi K, 2003. Comparative evaluation of ELISAs based on ten recombinant or purified leishmania antigens for the serodiagnosis of Mediterranean visceral leishmaniasis. Am J Trop Med Hyg 68: 312-320.

12. Sundar S, Reed SG, Singh VP, Kumar PCK, Murray HW, 1998. Rapid accurate field diagnosis of Indian visceral leishmaniasis. Lancet 351: 563-565.

13. Sundar S, Pai K, Sahu M, Kumar V, Marray HW, 2002. Immunochromatographic strip-test detection of anti-K39 antibody in Indian visceral leishmaniasis. Ann Trop Med Parasitol 96: 1923.

14. Bern C, Jha SN, Joshi AB, Thakur GD, Bista MB, 2000. Use of the recombinant $\mathrm{K} 39$ dipstick test and the direct agglutination test in a setting endemic for visceral leishmaniasis in Nepal. Am J Trop Med Hyg 63: 153-157.

15. Delgado O, Feliciangeli MD, Coraspe V, Silva S, Perez A, Arias J, 2001. Value of a dipstick based on recombinant rK39 antigen for differential diagnosis of American visceral leishmaniasis from other sympatric endemic diseases in Venezuela. Parasite 8: 355-357.

16. Jelinek T, Eichenlaub S, Löscher T, 1999. Sensitivity and specificity of a rapid immunochromatographic test for diagnosis of visceral leishmaniasis. Eur J Clin Mirobiol Infect Dis 18: 669670.

17. Zijlstra EE, Nur Y, Desjeux P, Khalil E, El-Hassan AM, Groen J, 2001. Diagnosing visceral leishmaniasis with the recombinant K39 strip test: experience from the Sudan. Trop Med Int Health 6: 108-113.

18. Carvalho SF, Lemos EM, Corey R, Dietze R, 2003. Performance of recombinant $\mathrm{K} 39$ antigen in the diagnosis of Brazilian visceral leishmaniasis. Am J Trop Med Hyg 68: 321-324.

19. Takagi H, Islam MZ, Itoh M, Islam MAU, Ekram ARMS, Hussain SM, Hashiguchi Y, Kimura E, 2007. Production of recombinant kinesin-related protein of Leishmania donovani and its 
application in the serodiagnosis of visceral leishmaniasis. $A m \mathrm{~J}$ Trop Med Hyg 76: 902-905.

20. Attar ZJ, Chance ML, el-Safi S, Carney J, Azazy A, El-Hadi M, Dourado C, Hommel M, 2001. Latex agglutination test for the detection of urinary antigens in visceral leishmaniasis. Acta Trop 78: 11-16.

21. Sarkari B, Chance M, Hommel M, 2002. Antigenuria in visceral leishmaniasis: detection and partial charecterisation of a carbohydrate antigen. Acta Trop 82: 339-348.

22. Islam MZ, Itoh M, Shamsuzzaman SM, Mirza R, Matin F, Ahmed I, Choudhury AKMS, Hossain MA, Qiu XG, Begam N, Furuya M, Leafasia JL, Hashiguchi Y, Kimura E, 2002. Diagnosis of visceral leishmaniasis by ELISA using urine samples. Clin Diagn Lab Immunol 9: 789-794.

23. Islam MZ, Itoh M, Mirza R, Ahmed I, Ekram ARMS, Sarder AH, Shamsuzzaman SM, Hashiguchi Y, Kimura E, 2004. Direct agglutination test with urine samples for the diagnosis of visceral leishmaniasis. Am J Trop Med Hyg 70: 78-82.

24. World Health Organization, 1996. Manual on Visceral Leishmaniasis Control. Geneva, Switzerland: World Health Organization.

25. World Health Organization, 1984. Leishmaniasis. Geneva, Switzerland: World Health Organization.
26. Khalil EAG, Zijlstra EE, Kager PA, El Hassan MA, 2002. Epidemiology and clinical manifestations of Leishmania donovani infection in two villages in an endemic area in eastern Sudan. Trop Med Int Health 7: 35-44.

27. Singh S, Kumari V, Singh N, 2002. Predicting kala-azar disease manifestations in asymptomatic patients with latent Leishmania donovani infection by detection of antibody against recombinant K39 antigen. Clin Diagn Lab Immunol 9: 568-572.

28. Jha TK, Sundar S, Thakur CP, Backmann P, Karbwang J, Fischer C, Vos A, Berman J, 1999. Miltefosine, an oral agent, for the treatment of Indian visceral leishmaniasis. N Engl J Med 341: 1795-1800.

29. Rijal S, Boelaert M, Regmi S, Karki BMS, Jacquet D, Singh R, Chance ML, Chappuis F, Hommel M, Desjeux P, Van der Stuyft P, Le Ray D, Koirala S, 2004. Evaluation of urinary antigen-based latex agglutination test in the diagnosis of kalaazar in eastern Nepal. Trop Med Int Health 9: 724-729.

30. Sundar S, Sing RK, Bimal SK, Gidwani K, Mishra A, Mauriya R, Singh SK, Manandar KD, Boelaert M, Rai M, 2007. Comparative evaluation of parasitology and serological tests in the diagnosis of visceral leishmaniasis in India: a phase III diagnostic accuracy study. Trop Med Int Health 12: 284-289. 